\title{
The Effects of Various Warm-Up Devices on Baseball Bat Velocity in Collegiate Baseball Players: A Pilot Study
}

\author{
Jordan L. Cola, Ph.D., CSCS \\ Department of Kinesiology \\ William Paterson University \\ 300 Pompton Rd. Wayne, NJ 07470, USA \\ East Stroudsburg University \\ 200 Prospect St. East Stroudsburg, PA, 18301 \\ Toni Lasala, Ph.D., CSCS, LMT \\ Department of Kinesiology \\ William Paterson University \\ 300 Pompton Rd. Wayne, NJ 07470, USA
}

\begin{abstract}
Purpose: The purpose of this study was to examine the effects of various weighted warm-up devices on standard baseball bat velocity and trajectory in collegiate baseball players. Methods: Three, right-handed (age 19.3 years \pm 1.5 years; height 1.74 meters \pm .13 meters; mass 81 kilograms \pm 20.4 kilograms; baseball experience 14.2 years \pm 1.3 years)volunteered. Maximal bat velocity was obtained by swinging the $30 \mathrm{oz}$ standard bat for the control condition. Participants were then instructed to perform a general and specific warm-up with each of the weighted bats (standard bat with $16 \mathrm{oz}$ donut ring (46oz total) and standard bat with $24 \mathrm{oz}$ power sleeve (54oz total)) on separate days. Following the warm-up procedures, participants were instructed to swing 3 times with the 30oz standard bat for maximal velocity while impacting the ball resting on the tee located belt-high and in the middle of home plate. Results: No significant differences were revealed by Shewart Chart method for baseball bat velocity. Conclusion: Based upon no changes in the dependent variable in the population tested, Division II collegiate athletes can choose any of the warm-up devices investigated because no deleterious effects were observed.
\end{abstract}

Keywords: Evenly distributed, bat velocity, exit velocity, complex training, post activation potentiation

\section{Introduction}

Baseball is a game which requires a mastery of many skills. Specifically, coordinated motor skills are needed to perform various tasks of the game such as fielding, throwing and hitting. Mastery of these skills is an essential prerequisite for the participants to possess to compete at the collegiate level, augment personal performance and theoretically increase the occurrence of a game win. Coaches design frequent practice sessions to address team needs as well as enhancing the skills of the individual player. During team practice sessions, a large percentage of time and effort is focused on training an effective baseball swing while simultaneously attempting to maximize bat velocity to theoretically improve hitting performance during live gameplay (Montoya, Brown, Coburn, \& Zinder, 2009).Bat velocity is commonly referred to as bat speed or swing velocity. However, regardless of the nomenclature that one uses, generation of maximal swing velocity at the instant of ball contact is an essential component to successful hitting. Increasing the speed at which a ball leaves a bat, or exit velocity, is a fundamental element in a successful hitting (Koenig, Mitchell, Hannigan, \& Clutter, 2004) and has quickly become one of the most popular baseball statistics. In fact, holding all other factors constant, exit velocity of the ball after impact is directly proportional to the velocity the baseball bat is traveling at impact (Koenig, Mitchell, Hannigan, \& Clutter, 2004). Baseball bat velocity is influenced by several factors. First, increased velocity of the bat is a factor regarding the ability of the hitter to appropriately position a bat both temporally and spatially during the swing after recognition of the type and location of the pitch (Koenig, Mitchell, Hannigan, \& Clutter, 2004).This represents the measure of how well the hitter can locate the bat to the proper place at the appropriate time to contact the thrown object. 
Secondly, bat velocity has a direct relationship to the speed at which the baseball is thrown toward the hitter. Fastball velocities at the college level range from 87-95 miles per hour, with an overall average of 91 miles per hour (NCSA). At this velocity, the batter has .4 seconds to react to the thrown baseball (Weiskopf, 1975). With this finite window of time for the hitter to react to the thrown object, increased bat velocity produced by the hitter is vital to contact the incoming ball traveling at such a high velocity. Once the hitter intentionally makes the decision to initiate the swing towards the incoming pitch, the velocity at which the bat is traveling becomes even more important. Maximum swing velocity meeting maximum ball velocity at the instant of contact will produce maximal force against the pitched baseball resulting in maximal exit velocity of the ball, post-contact, resulting in an increase in the distance traveled by the ball (Adair, 2002).

In an attempt to increase bat velocity and subsequently increase exit velocity and distance traveled by the ball postimpact, coaches, trainers and players suggest the use of various devices which can be added to the player's game bat during batting warm-ups. Players from the Little League level to those playing in Major League Baseball traditionally swing weighted bats in the on-deck circle as part of their warm-up prior to stepping in the batter's box to face live pitching. While the specific nature of the individual warm-up routines may fluctuate from hitter to hitter, the warm-up devices remain relatively consistent. Presently, amateur and professional baseball players commonly use a $16 \mathrm{oz}$ donut ring or $24 \mathrm{oz}$ PowerSleeve added to the hitter's game bat during warm-ups prior to stepping in the batters' box. The fundamental premise behind swinging an overweight warm-up bat in the on-deck circle prior to swinging a standard bat is that since motorunit recruitment follows a definitive sequence, additional motor units activated by the over-weighted warm-up device and may continue to function when the extra load is removed, resulting in increased bat velocity when swinging the less heavy, standard bat (DeRenne, Ho, Hetzler, \& Chai, 1992), thus subsequently augmenting performance for the hitter and team.

In contrast, it has been proposed that the dynamic wielding of an over-weighted device had no significant effect on post warm-up speed of movement, but only created a kinesthetic illusion of increased speed (DeRenne, Ho, Hetzler, \& Chai, 1992). It is clear that previous studies on the effects of weighted warm-up devices on bat velocity have shown inconsistencies. Warm-ups using heavier bats have been shown to produce increased swing velocities (Reyes \&Dolny, 2009), decreased swing velocities (Southard \& Groomer, 2003; Montoya et al., 2009; DeRenne, Ho, Hetzler, \& Chai, 1992), and unaltered swing velocities (Szymanski et al., 2011) as compared to standard baseball bats depending upon the study reviewed. Given the diversity of commercially available devices that a hitter can choose from to alter the weight of the bat during warm-up swings, along with discrepancies in the literature as to which device or the amount and/or location of the added weight produces the greatest post warm-up bat velocity, the question of 'Which warm-up device elicits the greatest post warm-up bat velocity?' remains unclear. Therefore, the purpose of this study was to provide an evidence-based comparison on the use of a custom made, evenly-distributed, overweight warm-up device to commercially available weighted devices while examining the effects of weighted warm-up devices on standard baseball bat velocity in collegiate baseball players.

\section{Methods}

\subsection{Subjects}

Three right-hand swinging participants volunteered for this pilot study (age 19.3 years \pm 1.5 years; height 1.74 meters \pm .13 meters; mass 81kilograms \pm 20.4 kilograms; baseball experience 14.2 years \pm 1.3 years). Anthropometric data is summarized in Table 1. Each of the participants provided written consent following a full explanation of the procedures, which were approved by the University Intuitional Review Board. Participants were accepted for the study if they met the following inclusion criteria: (1) Participants from Varsity baseball team, (2) College aged male within the range of 18-24 years of age, (3) free from musculoskeletal injury 8 months prior to the commencement of the study via the Health History injury and Par-Q forms.

\section{Table 1. Descriptive Statistics}

\begin{tabular}{llllll} 
& $\mathrm{N}$ & \multicolumn{1}{c}{ Min } & \multicolumn{1}{c}{ Max } & Mean & SD \\
\hline Age $(\mathrm{yr})$ & 3 & 18.00 & 21.00 & 19.5 & 1.5 \\
Mass $(\mathrm{kg})$ & 3 & 66.5 & 102.00 & 81.00 & 20.4 \\
Height $(\mathrm{m})$ & 3 & 1.23 & 1.86 & 1.54 & .13 \\
& & & & & \\
\hline
\end{tabular}

\subsection{Instrumentation}

An eight-camera (MX-40) motion analysis system (Vicon, Oxford, UK) was used sampling at $100 \mathrm{~Hz}$ to capture the swings performed by the participants. Vicon's Plug-In Gait model for retro-reflective marker placement was followed 
for all participants. The global reference frame was defined as the three-dimensional coordinate system where the movement of interest took place. Each of the three axes was perpendicular to each other. For this study, the positive Yaxis was the most critical because it was used to obtain the velocity of the baseball bat at the instant of bat-ball contact. It was defined as the direction from home plate to the pitching rubber. When looking at the positive Y direction, positive $\mathrm{Z}$ was defined as pointing superiorly and positive $\mathrm{X}$ was defined as pointing to the right. Dynamic calibration of the system was performed for 30 seconds resulting in an image error of $>2 \mathrm{~mm}$ for all cameras prior to data collection.

\subsection{General Design}

This study was conducted utilizing a single-subject, A-B-A design allowing the ability to observe changes only in the presence of the intervention (Portney and Watkins, 2008). Particularly, changes in the target behavior from baseline to post-test, where one can logically assume that any change in the post-test was due to the intervention. This particular design comes with well-established internal validity because of the highly improbable nature that confounding factors would coincidentally occur at the commencement and termination of the intervention (Portney and Watkins, 2008).

\subsection{Familiarization}

Once inclusion criteria have been met and all forms are properly filled out and signed, the participants had anthropometric measurements taken by the principle investigator, which were needed for Vicon software analysis. The specific anthropometric measurements are as follows: shoulder width, shoulder offset, elbow width, wrist width hand thickness, knee width, ankle width, leg length, height, and weight; which were obtained using anthropometric calipers and a spring-loaded tape measure. This familiarization session took place one day prior to data collection. The participants were asked to wear neoprene (spandex) baseball sliding shorts and a neoprene $t$-shirt for the familiarization session, as well as subsequent visits to the Biomechanics laboratory. Thirty-nine retro-reflective markers were placed on the participants at the specific anatomical locations following Vicon's Plug-In Gait model. On the locations of the body which were covered by clothing, the markers were attached via Velcro; for the parts of the body which were exposed, two-way tape was used to secure the marker to the anatomical landmark. The exposed locations were thoroughly cleaned with an alcohol pad to rid the location of body oils to allow the marker to be firmly attached to the skin.

\subsection{Warm-up Procedures}

The participants reported to the Biomechanics laboratory on the day of testing. Following the application of the markers, the participants were instructed to swing a standard, $30 \mathrm{oz}$ game bat 3 times for maximal velocity which served as the baseline or control condition. Following the control condition, after a 10 minute rest period, the participant performed a standardized general warm-up consisting of overhead and behind the back stretching with a randomly assigned warm-up device for a period of one minute (DeRenne, Ho, Hetzler, \& Chai, 1992). The warm-up devices were as follows: (1) a standard $33 \mathrm{in} / 30 \mathrm{oz}$ game bat serving as the control, (2) a weighted bat with a 16 ounce 'donut' slid onto the barrel of the standard $33 \mathrm{in} / 30 \mathrm{oz}$ baseball bat (46oz total), (3) a weighted bat with a 24 ounce Pow'r sleeve slid onto the barrel of the standard 33in/30oz baseball bat (54oz total), (4)a custom-made wooden baseball bat with weight evenly distributed along the length of the bat totaling $46 \mathrm{oz}$ and (5)a custom-made wooden baseball bat with weight evenly distributed along the length of the bat totaling $54 \mathrm{oz}$.

The order of the weighted bats were randomized and counterbalanced ensuring that participants used each bat over 5 days of data collection. Each session was 24 hours apart to minimize carryover effects. The procedures of DeRenne, Ho, Hetzler \& Chai, (1992) were strictly followed, with the only alterations being the custom weighted, evenly distributed baseball bats.

Following the general warm-up with a specific bat, the participants were then instructed to perform a specific warm-up which consisted of swinging a specific weighted device 4 consecutive times as fast as possible in a typical batting motion. Following the general and specific warm-up, the participant was then instructed to pick up the standard $33 \mathrm{in} / 30 \mathrm{oz}$ bat and swing it 2 times in a way that is comfortable to the participants.

\subsection{Experimental Procedures}

Following the 2 swings of the standard bat, the participant was then instructed to swing the standard $33 \mathrm{in} / 30 \mathrm{oz}$ bat 3 times while hitting a baseball off of a standard baseball hitting tee, with 20 seconds of rest between each swing. The baseball, which was supported by the tee, was located in an area which is consistent with a fastball down the middle of home plate. The height of the baseball was belt high, which is the location that is ideal for maximum contact with the ball. The above process will be repeated until all warm-up bats are utilized by the participants, with subsequent swings with the standard bat for each variation of the warm-up device. 


\subsection{Data Reduction and Analysis}

All kinematic data were smoothed using a generalized cross-validated quintic spline procedure (CVQSP) prior to further analyses.

Following the spline procedures, data was then exported to an excel spreadsheet for further analysis. Smoothed position data was then differentiated using the first central difference method to provide the linear velocity value of the marker located at the distal end of the barrel of the bat in the $\mathrm{Y}$ and $\mathrm{Z}$ axes. All analysis was performed using Microsoft Excel $^{\circledR}$.

\section{Results}

To find significant differences within the data, the Shewart Chart method, commonly referred to as the two-standard deviation method, was utilized to assess variability within the baseline phase by calculating the mean and standard deviation of data points within that phase (Portney and Watkins, 2008). Standard deviation was then added and subtracted from the mean to obtain the upper and lower limits, respectively. Significance is evident when a minimum of two consecutive data points' falls outside the upper and lower limits of the two standard deviation range.

\subsection{Participant 1 Velocity}

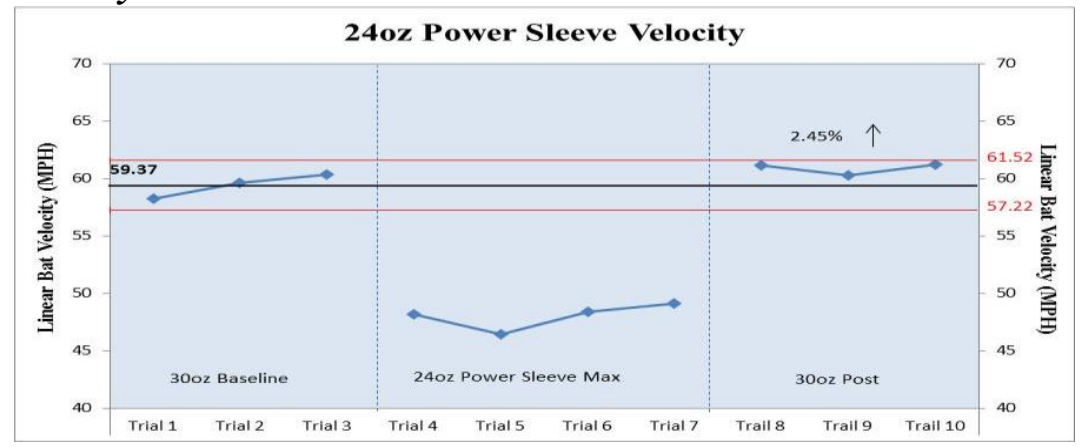

Figure 1. 24oz Power Sleeve Velocity. This graph illustrates the 24oz Power Sleeve velocity for the baseline, intervention and posttest. Also, the percent difference is illustrated in the posttest.

As seen in Figure 1, there was no significant difference between the baseline and the posttest phases, however a significant difference was observed when swinging the baseball bat with the additional weight added to the distal portion of the bat. Furthermore, an increase in velocity in the posttest of $2.45 \%$ was observed which equates to the participant swinging the bat $1.43 \mathrm{mph}$ faster than the baseline value of $59.37 \mathrm{mph}$, leaving a velocity of $60.8 \mathrm{mph}$ during the posttest when comparing to the baseline.

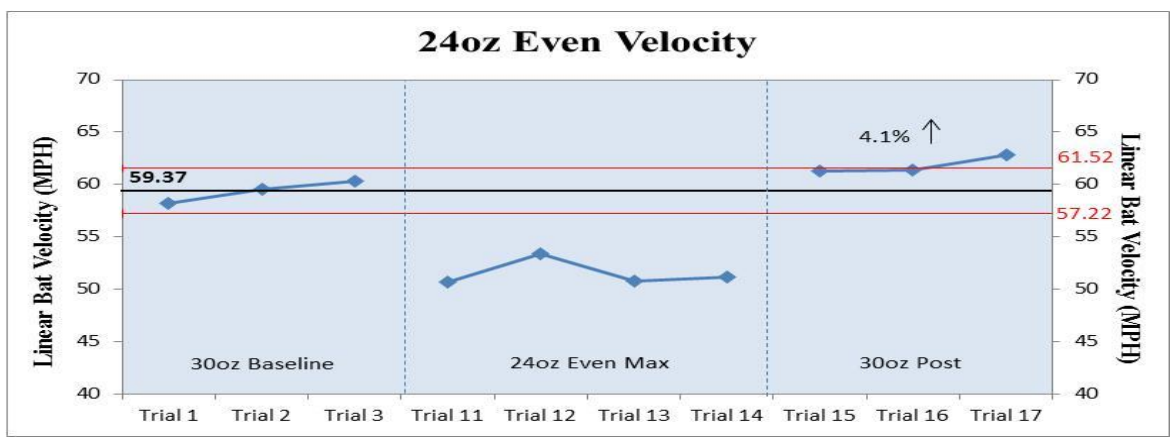

Figure 2. Participant 1 24oz Evenly Distributed Velocity. This graph illustrates the 240z Evenly Distributed velocity for the baseline, intervention and posttest. Also, the percent difference is illustrated in the posttest.

As seen in Figure 2, there was no significant difference between the baseline and the posttest phases, however a significant difference was observed when swinging the baseball bat with the additional weight added throughout the length of the custom wooden baseball bat. Furthermore, an increase in velocity in the posttest of $4.1 \%$ was observed which equates to the participant swinging the bat $2.43 \mathrm{mph}$ faster than the baseline value of $59.37 \mathrm{mph}$, leaving a velocity of $61.8 \mathrm{mph}$ during the posttest when comparing to the baseline. 


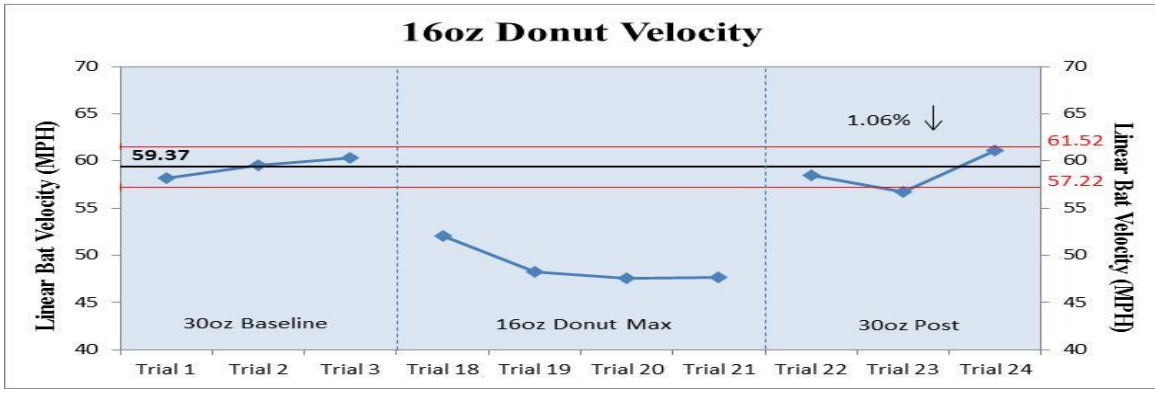

Figure 3. Participant 1 16oz Donut Velocity. This graph illustrates the $160 z$ donut velocity for the baseline, intervention and posttest. Also, the percent difference is illustrated in the posttest.

As seen in Figure 3, there was no significant difference between the baseline and the posttest phases, however a significant difference was observed when swinging the baseball bat with the additional weight added to the distal portion of the bat. Furthermore, a decrease in velocity in the posttest of $1.06 \%$ was observed which equates to the participant swinging the bat $.66 \mathrm{mph}$ slower than the baseline value of $59.37 \mathrm{mph}$ leaving a velocity of $58.71 \mathrm{mph}$ during the posttest when comparing to the baseline.

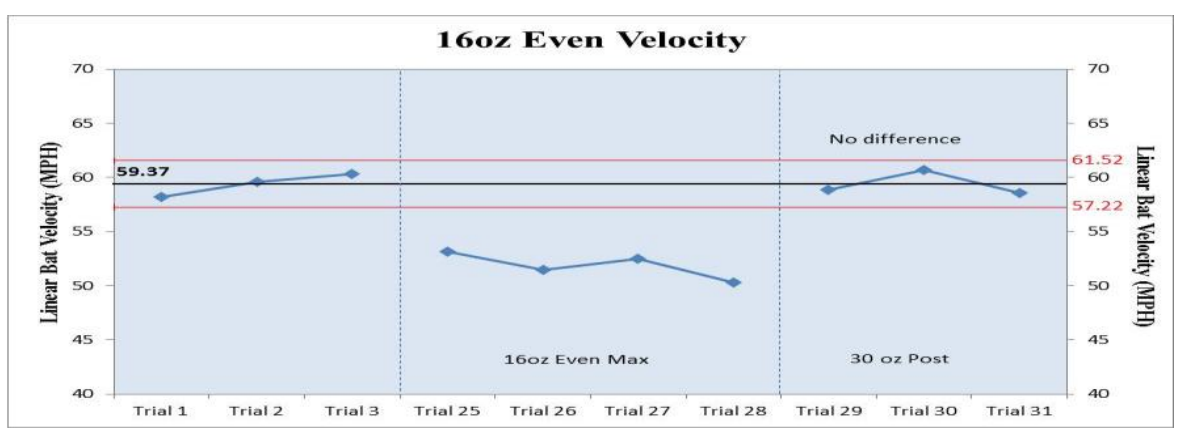

Figure 4. Participant 16oz Evenly Distributed Velocity. This graph illustrates the 16oz Evenly Distributed velocity for the baseline, intervention and posttest. Also, the percent difference is illustrated in the posttest.

As seen in Figure 4, there was no significant difference between the baseline and the posttest phases, however a significant difference was observed when swinging the baseball bat with the additional weight added throughout the length of the custom wooden baseball bat. Furthermore, there was no difference in percent difference, meaning during the posttest the participant was swinging the exact same velocity as was observed in the pretest when comparing to the baseline.

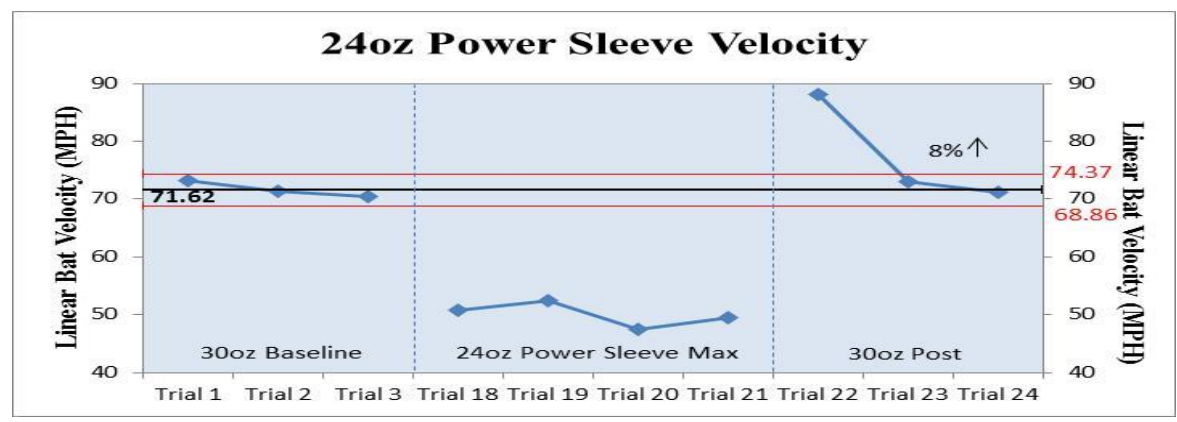

Figure 5. Participant 2 24oz Power Sleeve Velocity. This graph illustrates the 24oz Power Sleeve velocity for the baseline, intervention and posttest. Also, the percent difference is illustrated in the posttest.

\subsection{Participant 2 Velocity}

As seen in Figure 5, there was no significant difference between the baseline and the posttest phases, however a significant difference was observed when swinging the baseball bat with the additional weight added to the distal portion of the bat. Furthermore, an increase in velocity in the posttest of $8 \%$ was observed which equates to the participant swinging the bat $5.76 \mathrm{mph}$ faster than the baseline of $71.62 \mathrm{mph}$ leaving a velocity of $77.38 \mathrm{mph}$ during the posttest when comparing to the baseline. 


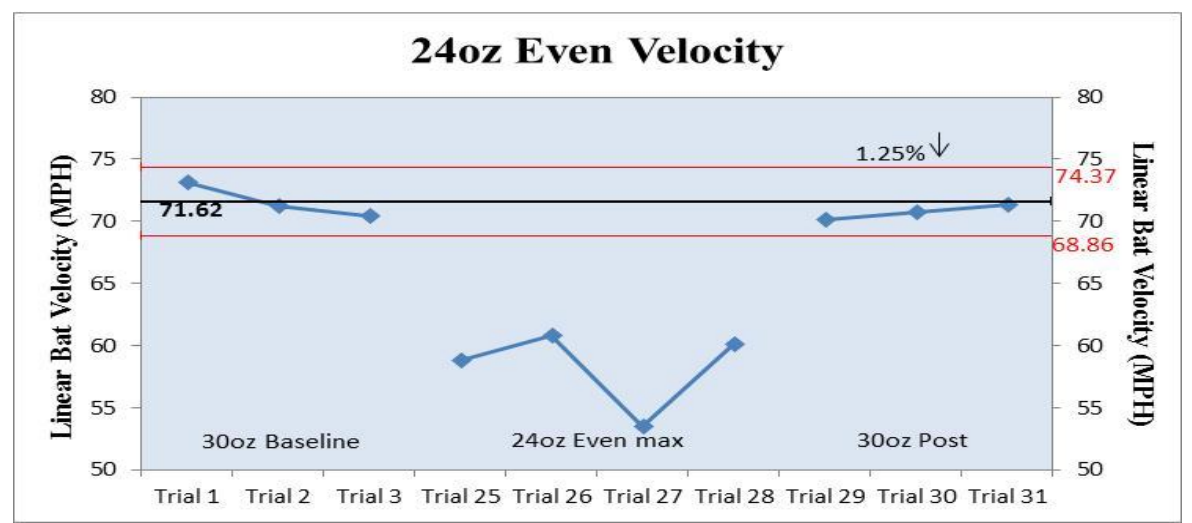

Figure 6. Participant 2 24oz Evenly Distributed Velocity. This graph illustrates the 24oz Evenly Distributed velocity for the baseline, intervention and posttest. Also, the percent difference is illustrated in the posttest.

As seen in Figure 6, there was no significant difference between the baseline and the posttest phases, however a significant difference was observed when swinging the baseball bat with the additional weight added throughout the length of the custom wooden baseball bat. Furthermore, a decrease in velocity in the posttest of $1.25 \%$ was observed which equates to the participant swinging the bat $.9 \mathrm{mph}$ slower than the baseline of $71.62 \mathrm{mph}$ leaving a velocity of $70.7 \mathrm{mph}$ during the posttest when comparing to the baseline.

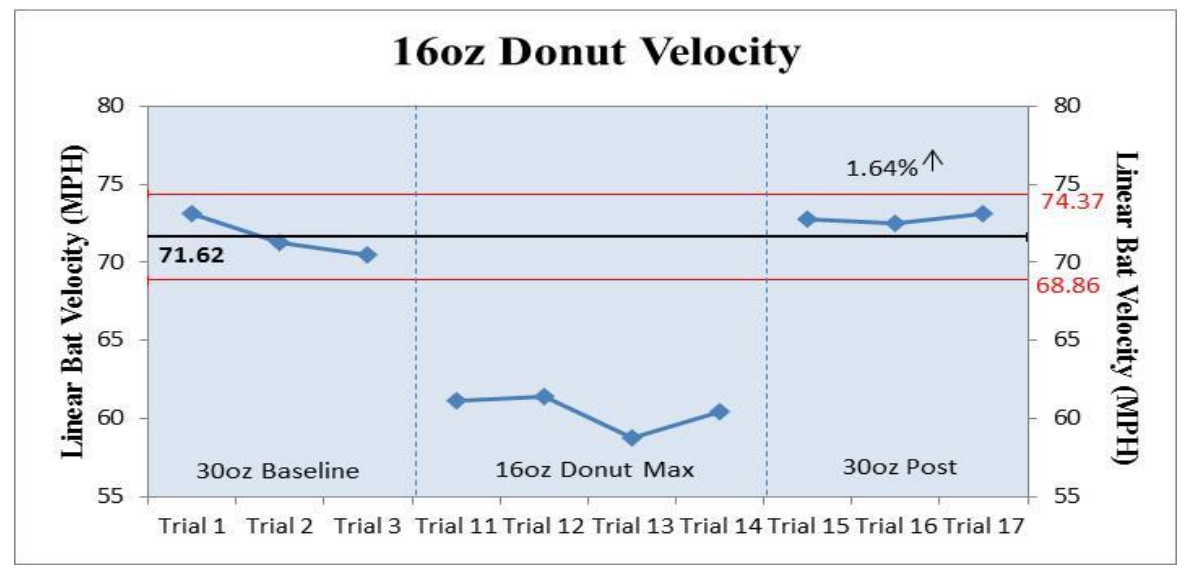

Figure 7. Participant 2 16oz Donut Velocity. This graph illustrates the $160 z$ donut velocity for the baseline, intervention and posttest. Also, the percent difference is illustrated in the posttest.

As seen in Figure 7, there was no significant difference between the baseline and the posttest phases, however a significant difference was observed when swinging the baseball bat with the additional weight added to the distal portion of the bat. Furthermore, an increase in velocity in the posttest of $1.64 \%$ was observed which equates to the participant swinging the bat $1.18 \mathrm{mph}$ faster than the baseline of $71.62 \mathrm{mph}$ leaving a velocity of $72.8 \mathrm{mph}$ during the posttest when comparing to the baseline.

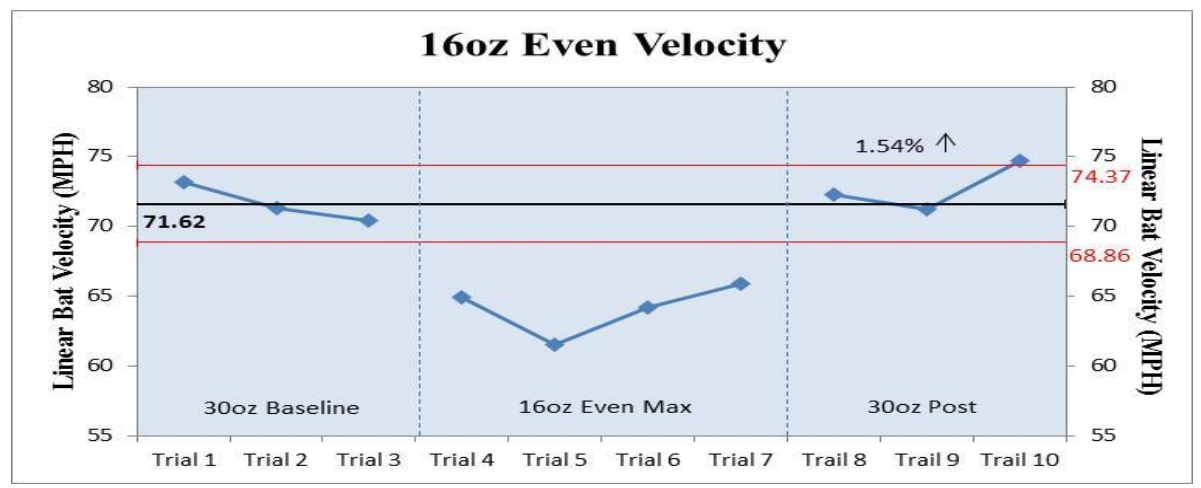


Figure 8. Participant 2 16oz Evenly Distributed Velocity. This graph illustrates the 16oz Evenly Distributed velocity for the baseline, intervention and posttest. Also, the percent difference is illustrated in the posttest.

As seen in Figure 8, there was no significant difference between the baseline and the posttest phases, however a significant difference was observed when swinging the baseball bat with the additional weight added throughout the length of the custom wooden baseball bat. Furthermore, an increase in velocity in the posttest of $1.54 \%$ was observed which equates to the participant swinging the bat $1.10 \mathrm{mph}$ faster than the baseline of $71.62 \mathrm{mph}$ leaving a velocity of $72.7 \mathrm{mph}$ during the posttest when compared to the baseline.

\subsection{Participant 3 Velocity}

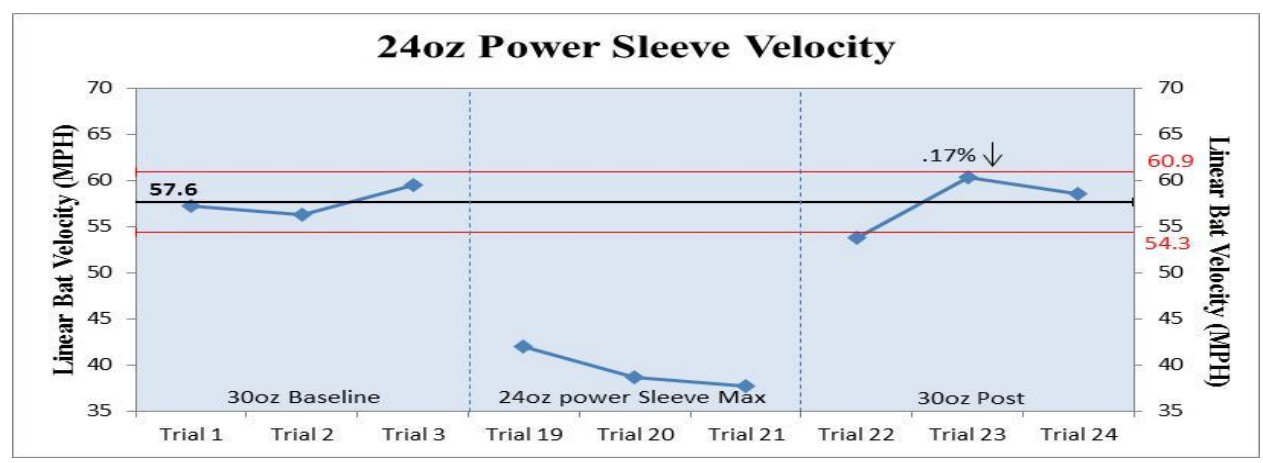

Figure 9. Participant 3 24oz Power Sleeve Velocity. This graph illustrates the $240 z$ Power Sleeve velocity for the baseline, intervention and posttest. Also, the percent difference is illustrated in the posttest.

As seen in Figure 9, there was no significant difference between the baseline and the posttest phases, however a significant difference was observed when swinging the baseball bat with the additional weight added to the distal portion of the bat. Furthermore, a decrease in velocity in the posttest of $.17 \%$ was observed which equates to the participant swinging the bat $.1 \mathrm{mph}$ slower than the baseline of $57.6 \mathrm{mph}$ leaving a velocity of $57.5 \mathrm{mph}$ during the posttest when comparing to the baseline.

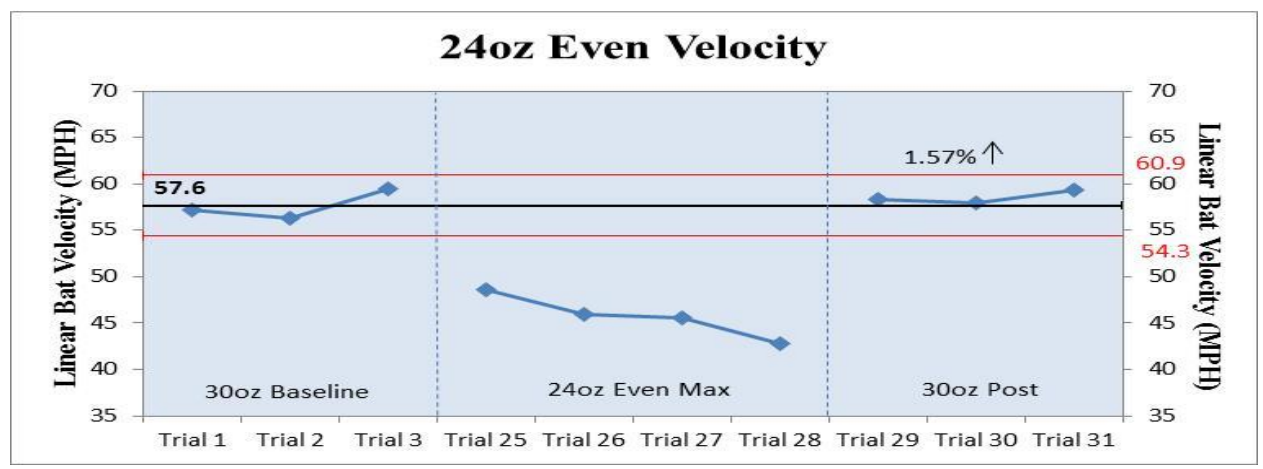

Figure 10. Participant 3 24oz Evenly Distributed Velocity. This graph illustrates the 24oz Evenly Distributed velocity for the baseline, intervention and posttest. Also, the percent difference is illustrated in the posttest.

As seen in Figure 10, there was no significant difference between the baseline and the posttest phases, however a significant difference was observed when swinging the baseball bat with the additional weight added throughout the length of the custom wooden baseball bat. Furthermore, an increase in velocity in the posttest of $1.57 \%$ was observed which equates to the participant swinging the bat $.86 \mathrm{mph}$ faster than the baseline of $57.6 \mathrm{mph}$ leaving a velocity of $58.51 \mathrm{mph}$ during the posttest when comparing to the baseline. 


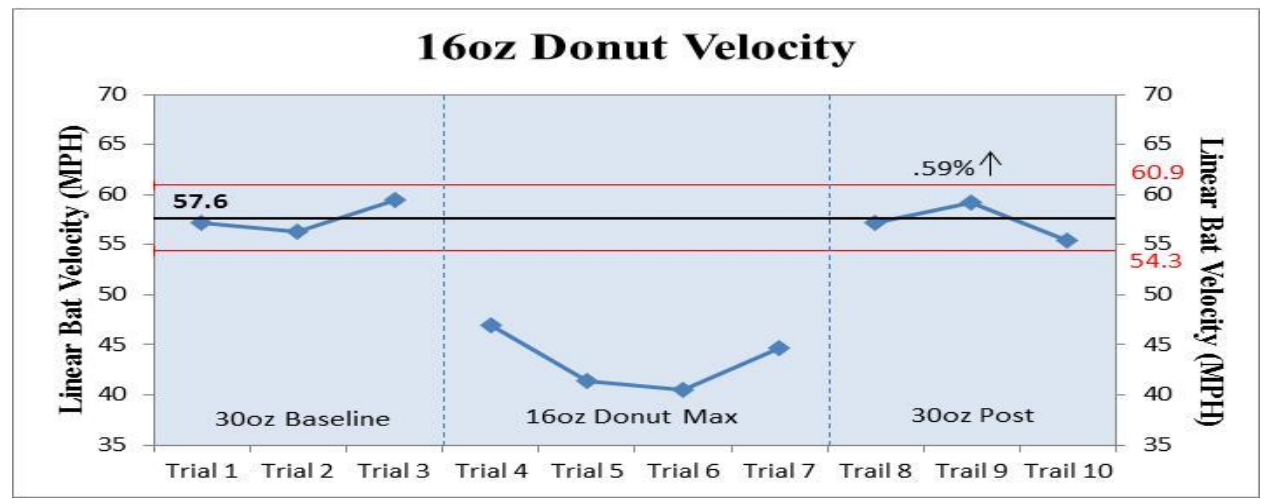

Figure 11. Participant $3 \mathrm{16oz}$ Donut Velocity. This graph illustrates the $160 z$ donut velocity for the baseline, intervention and posttest. Also, the percent difference is illustrated in the posttest.

As seen in Figure 11, there was no significant difference between the baseline and the posttest phases, however a significant difference was observed when swinging the baseball bat with the additional weight added to the distal portion of the bat. Furthermore, an increase in velocity in the posttest of .59\% was observed which equates to the participant swinging the bat $.34 \mathrm{mph}$ faster than the baseline of $57.6 \mathrm{mph}$ leaving a velocity of $57.94 \mathrm{mph}$ during the posttest when comparing to the baseline.

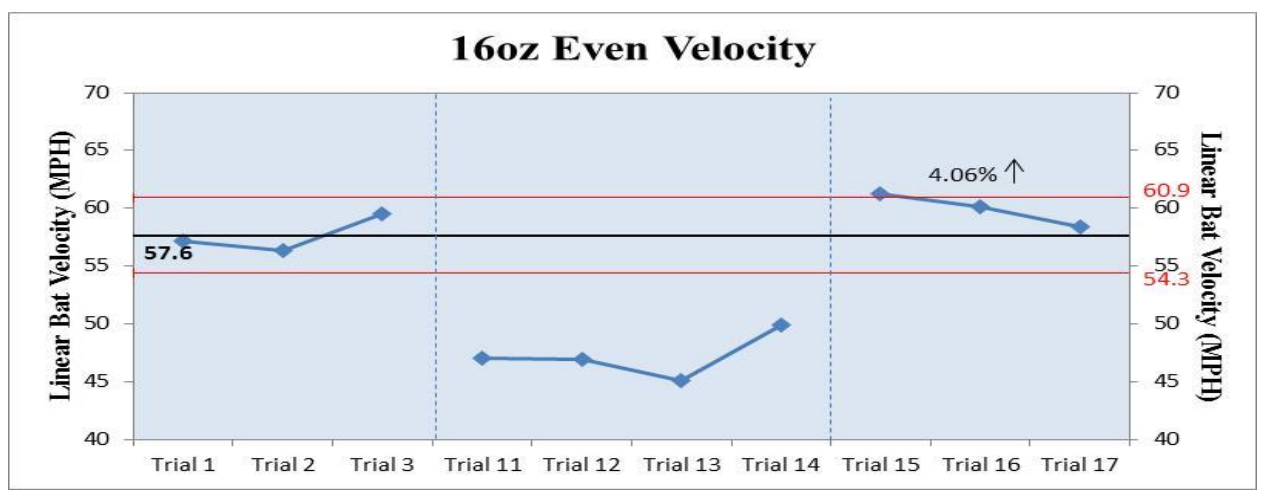

Figure 12. Participant 3 16oz Evenly Distributed Velocity. This graph illustrates the 16oz Evenly Distributed velocity for the baseline, intervention and posttest. Also, the percent difference is illustrated in the posttest.

As seen in Figure 12, there was no significant difference between the baseline and the posttest phases, however a significant difference was observed when swinging the baseball bat with the additional weight added throughout the length of the custom wooden baseball bat. Furthermore, an increase in velocity in the posttest of $4.06 \%$ was observed which equates to the participant swinging the bat $2.34 \mathrm{mph}$ faster than the baseline of $57.6 \mathrm{mph}$ leaving a velocity of 59.94mph during the posttest when compared to the baseline.

\section{Discussion}

\subsection{Participant 1}

The purpose of this study was to examine the effects of various weighted warm-up devices on standard baseball bat velocity in collegiate baseball players; specifically, to compare custom made, evenly-distributed weighted bats to commercially available devices. The main finding within the results of participant one agrees with previous research conducted by Szymanski et al., (2011), DeRenne, Ho, Hetzler, \& Chai, (1992), Montoya et al. (2009), Otsuju, Abe, and Kinoshita, (2002) and Southard and Groomer (2003)

Which revealed that the most commonly used warm-up device throughout all levels of play, the 16oz donut ring, produced slower bat velocities (Fig. 3).However, these data disagree with Szymanski et al., (2011), DeRenne, Ho, Hetzler, \& Chai, (1992) for the sole reason that an increase in bat velocity was observed after dynamically wielding the heavier 24oz power sleeve (Fig.1).Observing higher velocities while swinging the heavier bat could possibly be explained by Henneman's size principle of motor unit recruitment. This principle states that the size of the recruited motor unit increases with the level of tension at which it is recruited, in other words, the smallest diameter motor unit is recruited first and the largest unit last (Henneman, 1974a). 
When looking at the percent difference results for the bats with the additional 16oz weight (Fig. 3), one could postulate that the size principle wasn't utilized to its fullest potential. It's plausible that only Type I and IIa fibers were recruited which would produce less force applied to the bat resulting in less power and speed of the swing than if the higher force producing Type IIx were also recruited. It is possible that by not having a load great enough to elicit the recruitment by the CNS of those higher force producing fiber types, a decrease in swing has been observed after dynamically wielding the additional $16 \mathrm{oz}$ weighted bats when compared to the additional $24 \mathrm{oz}$ weighted bats.

An explanation as to why the increase of velocity was observed after dynamically wielding the $24 \mathrm{oz}$ weighted bats (Fig. 1\&2) may be explained by increasing motor unit recruitment, specifically Type IIx, due to the increased intensity of the additional weight resulting in increased activation from the CNS, thus increasing force and speed applied to the bat. Additionally, previous studies have shown that preloading a muscle can augment force production and motor unit recruitment (Tillin \& Bishop, 2009; Kamimura \& Takenaka, 2007). When dynamically wielding the more massive bat, there may have been an increase in muscle sensitivity to the preload resulting in postactivation potentiation (PAP). The fundamental belief surrounding PAP is that preceding heavy loading encourages increased central nervous system (CNS) stimulation, which results in greater motor unit recruitment and force, with residual effects lasting up to thirty minutes (Chiu, Fry, Weiss, et al., 2003; Rixon, Lamont, \& Bemden, 2007).

A study conducted by Hamada et al. (2000a) showed that PAP is most effective when Type II fibers are at a greater percentage of the muscles being used. Thus, this phenomenon can be correlated to an increased performance in athletes who rely on a more rapid twitch contraction time for optimal athletic performance in explosive activities such as sprinting, jumping, throwing and hitting (Horwath \& Kravitz, 2008); further solidifying the postulation that the 24oz weighted bats elicited a greater number of Type IIx fiber types resulting in an increased swing velocity post warm-up when comparing to the $16 \mathrm{oz}$ weighted bats because of an increase in preload. Additionally, one my postulate that with the additional mass the 24ozPower Sleeve provides an increased isometric contraction, via increased inertia, resulting in more preload of the triceps which may lead to increased muscle responsiveness and number of cross bridge attachments within the muscle. This would result in more work and kinetic energy applied to the bat throughout the swing, resulting in the increased velocity observed with the $24 \mathrm{oz}$ device because preloading is central in the development of strength early in the movement, especially at high velocities (Baechle \& Earle, 2008).

Finally, Coactivation is the mechanical effect of making a joint stiff and difficult to perturb which opposes the notion of reciprocal activation. Reciprocal activation is defined as the simultaneous activation of muscle(s) with a mechanical action on a joint (agonist) and inhibition of muscles with the opposite mechanical action (antagonists) (Binder \& Hirokawa, 2009). One may postulate that during the $16 \mathrm{oz}$ conditions, the increase in muscle and joint stiffness may have resulted from activation from the CNS with the cerebellum playing a central role in substituting reciprocal activation for coactivation (Kjaer, Krogsgaard, Magnusson, et al., 2008). Thus, possibly decreasing the speed of the swing when compared to the $24 \mathrm{oz}$ conditions where PAP was predominate due to the excitation of the Group Ia afferent muscle nerves enhancing the H-reflex, rather than an inhibition during the $16 \mathrm{oz}$ swings causing a coactivation resulting in a slower swing. Kauffman and Greenisen, (1973) stated the magnitude of the biceps brachii involvement, following the use of a weighted bat, could abolish the advantage of additional involvement of the triceps brachii motor unit. Being that there was an increase in post warm-up velocity after dynamically wielding the more massive $24 \mathrm{oz}$ bat, one could postulate that there is a threshold switching from coactivation to PAP. It is possible that the load was too light in the $16 \mathrm{oz}$ conditions resulting in excluding the higher force producing Type IIx fiber types and exhibiting an inhibition of Group 1a afferents, thus not providing an appropriate environment to exhibit a PAP effect. However, once the load was increased, the appropriate fiber types were recruited, and excitation of the Group 1a afferents occurred providing an appropriate environment to exhibit a PAP and thus an increase in swing velocity.

\subsection{Participant 2}

With regards to fiber type and PAP, similar results were observed for participant 2. Although significant differences were not observed an increase in velocity was detected. The Power Sleeve (Fig 5.), donut (Fig 7.) and the 16oz evenly distributed bat (Fig. 8) showed an increase in velocity, leading to the postulation of an appropriate environment to induce a PAP and a decrease in coactivation to increase the swing velocity. Furthermore, participant 2 possessed greater lean body mass which was visually observed from a larger cross sectional area of the upper extremities when comparing to the other two participants. This larger, visible amount of lean body mass may have resulted in the predominant fiber type being Type IIx fast twitch fibers, thus further enhancing the environment to exhibit a PAP effect and excitation of the H-reflex to increase swing velocity. However, a decrease in velocity was observed for the $24 \mathrm{oz}$ evenly distributed bat (Fig. 6). 
High levels of resistance training could have benefits, such as increasing the speed of the swing via fiber type transitions (Type IIa to Type IIx), increased recruitment of fast twitch type IIx motor units via selective recruitment or neural adaptations such as increased reflex potentiation (Behm, 1995). However, the decrease in velocity may have been a result of the participant not fully complying with the studies explicit instructions to refrain from physical activity prior to testing which could have fatigued the muscles prior to performing the swings. It has been shown that resistance training decreases the coactivation response. In study conducted by Behm, (1995), trained individuals had a lesser amount of coactivation prior to fatigue but significantly greater amount coactivation following fatigue. Behm, (1995) claimed that the increased coactivation succeeding fatigue may be connected to the finding that trained individuals also had enhanced muscle activation and it's possible that supraspinal neural drive following fatigue results in more dispersion of the signals to the antagonist muscle, which may have played a role in decreasing the speed of the swing.

\subsection{Participant 3}

Much like the previous participants, participant 3 showed increases in velocity. Increases were observed for the $24 \mathrm{oz}$ evenly distributed (Fig. 10), 16oz donut (Fig.11) and 16oz evenly distributed conditions (Fig.12). Possible reasons for the increase in velocity under these three conditions may be due to the excitation of the H-reflex, decrease of coactivation of the antagonist and agonist muscles, as well as a proper environment to exhibit a PAP, as was previously mentioned. However, a decrease in velocity was observed after dynamically wielding the 24oz Power Sleeve (Fig. 9). Moment of Inertia (MOI), the angular equivalent of inertia, is the measure of the resistance that an object exerts to a change in its motion about a specified axis (Enoka, 2008). Being that the axis of rotation is located near the hands when the participants is holding the bat, MOI is increased as the mass is located farther away from the axis of rotation. MOI is not only a function of mass, but more importantly mass distribution. In fact, Fleisig, Zheng, Stodden, \& Andrews (2002) stated that swing velocity had a stronger relationship with bat MOI than mass because it is likely to have bats with the absolute mass but varying MOI depending upon the location of the mass.

DeRenne et al., (1992) reported that swing velocity is reduced following a warm-up with a bat weighing more than $\pm 10 \%$ of the standard bat weight $(30 \mathrm{oz})$. The authors theorized that when bat weight was augmented by more than $10 \%$, there may be changes in hitting mechanics that would explain the reduction in swing velocity. Southard and Groomer, (2003) stated, with the use of dynamic systems terminology, that coordination is reorganized when the bat's MOI is scaled to a critical factor. A clue regarding the critical value required to change the swing pattern may lie in the findings of DeRenne et al., (1992) with decreases in velocity occurring when the device to be dynamically wielded exceeds the $\pm 10 \%$ range. As one can observe in the results, participant 3 may have been well within his critical factor to increase velocity (Fig. 10-12). However, when the critical factor exceeded what the hitter was able to handle, a decrease in velocity was observed with the more massive $24 \mathrm{oz}$ power sleeve (Fig. 9). This may lead to an inflation of the critical factor for this participant. All devices utilized by participant 3 were above the $\pm 10 \%$ threshold which DeRenne et al., (1992) suggests, however increases in velocity were observed (Fig 10-12). With the use of the evenly distributed $24 \mathrm{oz}$ bat, which had the same mass but different MOI, there was an increase in velocity which contradicts the results of the $24 \mathrm{oz}$ power sleeve (Fig. 9). This finding supports the results by Fleisig, Zheng, Stodden, \& Andrews (2002), in that, MOI has a stronger relationship with swing velocity than overall bat mass and by manipulating, or decreasing, the MOI while still weighting the bat, the critical factor may be increased above the $\pm 10 \%$ range providing an appropriate device to increase post warm-up velocity.

\section{Conclusion}

Much like Reyes and Dolny, (2009), this study found no significant differences in velocity, however increases in post warm-up velocity was observed which cannot go unrecognized. For every 1 mile per hour increase in swing velocity, a distance of 8 feet of flight time is added to the ball post impact which may result in a routine fly ball exceeding the distance of the fence resulting in a home run. This may directly affect the outcome of the game while at the same time increasing the performance of the participant (slugging percentage, batting average, etc.).

When comparing the results of the current study to those of DeRenne et al., (1992) which utilized high school participants with similar conditions and the same procedures, level of play seems to play a vital role. Participants possessing greater experience, improved hitting mechanics and increased strength showed a greater increase in post warm-up velocity. The older, stronger, more experienced participants may be able to overcome the inertia added to the bat thus resulting in a greater post warm-up effect which contradicts most studies investigating post warm-up velocity immediately following a weighted intervention. It seems that as level of play increases, the critical value of $\pm 10 \%$ of the standard bat weight proposed by DeRenne et al., (1992) and further substantiated by Southard and Groomer (2003) is also increased resulting in no detrimental effects to swing velocity. With the increase of the critical value, the bat may be loaded with more weight to allow the participant to take advantage of the PAP effect to the muscles while not severely sacrificing velocity when swinging during a game situation. This allows the participant more freedom to select 
an appropriate device to maximize post warm-up velocity. Based upon the results of this study, division two athletes can choose any one of the warm-up devices investigated because no deleterious effects were observed.

\section{References}

Adair, R.K. (2002). The Physics of Baseball (3rd ed.). New York: Harper Publishers.

Baechle, T. R., \& Earle, R. W. (2008). Essentials of strength training and conditioning. Human kinetics.

Behm, D.G. (1995). Neuromuscular implications and applications of resistance training. The Journal of Strength and Conditioning Reseach, 9(4), 264-274.

Binder, M. D., \& Hirokawa, N. (Eds.). (2009). Encyclopedia of neuroscience (Vol. 3166). Berlin, Heidelberg: Springer.

Chiu, L. Z., Fry, A. C., Weiss, L. W., Schilling, B. K., Brown, L. E., \& Smith, S. L. (2003). Postactivation potentiation response in athletic and recreationally trained individuals. The Journal of Strength \& Conditioning Research, 17(4), 671-677.

DeRenne, C., Ho, K.W., Hetzler, R.K., and Chai, D.X. (1992). Effects of warm up with various weighted implements on baseball bat swing velocity. Journal of Applied Sport Science Research, 6, 214-218.

Enoka, R.M. (2008). Neuromechanics of human movement. Human Kinetics.

Fleisig, G. S., Zheng, N., Stodden, D. F., \& Andrews, J. R. (2002). Relationship between bat mass properties and bat velocity. Sports Engineering, 5(1), 1-8.

Hamada, T., Sale, D.G., MacDougall, J.D., \& Tarnopolsky, M.A. (2000a). Postactivation potentiation, muscle fiber type, and twitch contraction time in human knee extensor muscles. Journal of Applied Physiology, 88, 21312137.

Henneman, E. (1974). "Organization of the Spinal Cord." Medical Physiology, 1, 13th ed., V.B. Monetcastle, Ed. (C.V. Mosby, St. Louis, MO).

Horwath, R., \& Kravitz, L. (2008). Postactivation potentiation: A brief review. IDEA Fitness Journal, 5(5), 21-23.

Kamimura, T., \&Takenaka, T. (2007). Potentiation of knee extensor contraction by antagonist conditioning contraction at several intensities. Journal of physiological anthropology, 26(4), 443-447.

Kauffman, I. B., \&Greenisen, M. C. (1973). Analysis of the validity of warm up with a weighted baseball bat. Paper presented at the annual meeting of the American Society of Mechanical Engineers, Detroit, MI.

Kjaer, M., Krogsgaard, M., Magnusson, P., Engebretsen, L., Roos, H., Takala, T., \& Woo, S. L. (Eds.). (2008). Textbook of sports medicine: Basic science and clinical aspects of sports injury and physical activity. John Wiley \& Sons.

Koenig, K., Mitchell, N. D., Hannigan, T. E., \& Clutter, J. K. (2004). The influence of moment of inertia on baseball/softball bat swing speed. Sports Engineering, 7(2), 105-117.

Montoya, B.S, Brown, L.F., Coburn, J.W., and Zinder, S.M. (2009). Effect of warm-up with different weighted bats on normal baseball bat velocity. Journal of Strength and Conditioning Research, 23, 1566-1569.

National Collegiate Scouting Association. 2002-2013. Baseball Pitcher. Retrieved from http://www.ncsasports.org/recruiting-tools/college-baseball-recruiting/baseball-recruiting-guidelines/baseballpitcher

Otsuju, T., Abe, M., and Kinoshita, H. (2002). After-effects of using a weighted bat on subsequent swing velocity and heaviness. Perceptual Motor Skills, 94, 119-126.

Portney, L. G., \& Watkins, M. P. (2008). Foundations of clinical research: applications to practice (Vol. 3). Upper Saddle River, NJ: Prentice Hall.

Reyes, G. F., \&Dolny, D. (2009). Acute effects of various weighted bat warm-up protocols on bat velocity. Journal of Strength and Conditioning Research, 23, 2114-2118.

Rixon, K. P., Lamont, H. S., \&Bemben, M. G. (2007). Influence of type of muscle contraction, gender, and lifting experience on postactivation potentiation performance. The Journal of Strength \& Conditioning Research, 21(2), 500-505.

Southard, D., \& Groomer, L. (2003). Warm-up with baseball bats of varying moments of inertia: Effect on bat velocity and swing pattern. Research quarterly for exercise and sport, 74(3), 270-276.

Szymanski, D. J., Beiser, E. J., Bassett, K. E., Till, M. E., Medlin, G. L., Beam, J. R., \&DeRenne, C. (2011). Effect of various warm-up devices on bat velocity of intercollegiate baseball players. The Journal of Strength \& Conditioning Research, 25(2), 287-292.

Tillin, N. A., \& Bishop, D. (2009). Factors modulating post-activation potentiation and its effect on performance of subsequent explosive activities. Sports medicine, 39(2), 147-166.

Weiskopf, D. (1975). Eyes have it. Athletic Journal, 55( 9), 18 -20; 72-76; 78-79. 